\title{
General Psychiatry Psychometric properties of the Caregiver Strain Questionnaire among Chinese parents of children with ADHD or ASD
}

\author{
Runxu Yang (D) , ${ }^{1}$ Huizhi Zhou, ${ }^{2}$ Jin Liu, ${ }^{3}$ Yufeng Wang, ${ }^{3}$ Yan Zhang, ${ }^{4}$ \\ Yanjiao Wang, ${ }^{1}$ Ruixiang Liu, ${ }^{5}$ Xia Cao, ${ }^{6}$ Dong Han, ${ }^{1}$ Shuran Yang, ${ }^{2}$ \\ Jianzhong Yang, ${ }^{4}$ Chuanyuan Kang ${ }^{2}$
}

To cite: Yang R, Zhou H, Liu J, et al. Psychometric properties of the Caregiver Strain Questionnaire among Chinese parents of children with ADHD or ASD. General Psychiatry 2021;34:e100246. doi:10.1136/ gpsych-2020-100246

$\mathrm{RY}$ and $\mathrm{HZ}$ contributed equally.

Received 19 April 2020

Revised 19 December 2020 Accepted 01 February 2021

\section{Check for updates}

(c) Author(s) (or their employer(s)) 2021. Re-use permitted under CC BY-NC. No commercial re-use. See rights and permissions. Published by BMJ.

For numbered affiliations see end of article.

Correspondence to Dr Chuanyuan Kang; kangba098@163.com

Dr Jianzhong Yang; jzhyang2004@163.com

\section{ABSTRACT}

Background There is an urgent need in clinical practice to measure the stress of parenting. The Caregiver Strain Questionnaire (CGSQ) was found to be useful to measure parenting stress, but it has not been validated among the Chinese population.

Aims To assess the reliability and construct validity of the Chinese version of CGSQ among Chinese parents.

Methods From 2016 to 2017, 266 parents (patient group) with a child having DSM-5-defined attention deficit hyperactivity disorder (ADHD) $(n=107)$ or autism spectrum disorder (ASD) $(n=159)$ and 268 parents of healthy children (control group) were recruited to the present study in Kunming, Yunnan province. All the parents were asked to fill out the Chinese version of CGSQ. We conducted exploratory factor analysis and confirmatory factor analysis (CFA) to verify construct validity of CGSQ in both patient and control groups. Cronbach's $\alpha$ coefficient as an index of internal consistency was assessed for each subscale. Fourteen days later, 23 subjects filled out the scale again. Intra-class correlation coefficient was calculated to evaluate the test-retest reliability.

Results (1) Cronbach's alpha of the global scale was 0.901 for the control group and 0.952 for the patient group. The test-retest reliability for the whole scale was 0.890; (2) CFA indicated that the three-factor model had better fitting indices compared with the two-factor mode in both groups. Besides, the fitting indices in the patient group were more favourable than those of the control group, with $\chi^{2} / \mathrm{df}=1.564$, Goodness-of-Fit Index $=0.841$, Comparative Fit Index $=0.954$, and root mean square error of approximation $=0.065$ for the patient group at threefactor model; (3) The caregiver strain of ASD parents was statistically higher than that of ADHD parents, and caregiver strain of ADHD parents was higher than that of control group.

Conclusion These findings provide initial evidence to support the construct validity and reliability of CGSQ as a parenting stress measurement tool for Chinese parents, especially for parents of children with ADHD or ASD.

\section{INTRODUCTION}

Parenting stress or caregiver strain can be defined as a "negative psychological reaction to the demands of being a parent". 1 Abidin $^{2}{ }^{3}$ asserted that parenting stress is created by a contradiction between the demands of parenting and personal resources that are used to meet those demands. Raising children with medical conditions, developmental or psychiatric disorders is oftentimes stressful, as those children often have special needs that can intervene in both individual and family functioning. Moreover, parents of children with emotional and behavioural problems often experience higher levels of parenting stress than parents of typically developed children. ${ }^{45}$ It is well understood that sometimes those responsibilities can be too burdensome for caregivers and can result in stress, which may lead to interrupted work, loss of personal time, worry and fatigue. ${ }^{3}$ Caregiver strain can have adverse consequences on the long-term welfare of both parents and their children. ${ }^{4}$ For example, parenting stress augments the risk of caregivers' poor mental health ${ }^{6}$, lack of parenting practices ${ }^{7}$ and hardship in conducting behavioural interventions for their children. ${ }^{8}$ While most of the research related to parenting stress focuses on parents having children with mental illness, parents with a typically developed child (or children) in mainland China are also worthy of note. With increasing urbanisation and industrialisation, aggravation of social competition, combined with the particularity of the onechild policy, parents often exhibit increasing expectations for their children's academic performance and occupational attainment, which may lead to a gradual increase in parenting stress. ${ }^{9} 10$

Among the various reasons for caregiver stress, a child's education may be one of the heaviest burdens. As reported from a recent survey conducted by Hong Kong and 
Shanghai Banking Corporation Limited (HSBC), "With the rise of the information economy and a challenging job market for young people, education has never been more important than it is today". ${ }^{11}$ The survey also revealed that as high as $93 \%$ of parents (943 parents from mainland China, including 504 from the Pearl River Delta region) were paying for private tuition for their children or have done so in the past, which ranks first among 15 investigated countries/regions. At the same time, Chinese parents are the most prepared, with over half $(55 \%)$ funding their children's education through general saving, investments or insurance. Parents of mainland Chinese children are furthermore most likely to make personal sacrifices for their children to succeed by reducing or completely stopping leisure activities/holidays. Indeed, there is an urgent need to study caregiver strain in the general population in order to improve the functioning of children and their families.

In order to investigate caregiver strain or parenting stress, several questionnaires have been developed worldwide. $^{12}{ }^{13}$ In mainland China, no domestic scale has been developed until now, but several scales have been imported from western countries and are frequently used to assess parenting stress. These include the Parenting Stress Index/Long Form (PSI-LF), Parenting Stress Index/Short Form (PSI-SF) and Caregiver Strain Questionnaire (CGSQ). However, none of these three questionnaires has been validated in mainland China.

The CGSQ was developed by Brannan et alin late $1997^{14}$, and was developed to measure levels of stress experienced by caregivers who are taking care of children or adolescents with emotional or behavioural disorders. In 2001, Peking University Sixth Hospital introduced the CGSQ into China, and the translator was a child psychiatrist with years of study experience abroad. Two senior experts who had many years of clinical experience in child psychiatry reviewed the Chinese version of the scale and concluded that it reflected the content of the original scale with high fidelity.

Several sections of the CGSQ are used to evaluate parenting stress: The Objective Caregiver Strain (OCGS), and the Subjective Caregiver Strain (SCGS) which can be divided into two subscales: The Externalized Subjective Caregiver Strain (ESCGS) and the Internalized Subjective Caregiver Strain (ISCGS) according to the original theoretical hypothesis of the founder. Brannan proposed two structural models for the CGSQ: two-factor model and three-factor model. The two-factor model includes two subscales: OCGS and SCGS, whereas the three-factor model consists of three subscales: OCGS, ESCGS and ISCGS. Comparison of fit indices between the two models showed that the three-factor model has better psychometric properties. ${ }^{1415}$

CGSQ has been used by caregivers of individuals with a variety of diagnoses. ${ }^{16}$ Until now, only a few studies demonstrated good internal consistency, reliability and construct validity of the CGSQ among different samples. ${ }^{151718}$ Compared with PSI-LF and PSI-SF, the
CGSQ is less time-consuming, consisting of only 21 items, which takes only $3-5$ min to complete, while the total item numbers is 120 for PSI-LF and 36 for PSI-SF.

To the best of our knowledge, there is no study so far that has explored whether or not the construct validity of the CGSQ fits the original theoretical hypothesis in a mainland Chinese population. Therefore, the present study aims mainly to investigate construct validity of CGSQ by exploratory factor analysis (EFA) and confirmatory factor analysis (CFA) in parents with a child diagnosed with either ADHD or ASD. Given that we also critically need a tool to assess parenting stress in the general population and the term description of CGSQ is relatively general and not specific to any disease, we would test the psychometric properties of CGSQ in parents with typically developing children. Another aim is to compare the three-factor and two-factor models in both groups (ie, patients and a healthy population). Finally, we have interest in exploring how the CGSQ differentiates levels of parenting stress among parents with a healthy child, or a child of ADHD or ASD.

\section{METHODS}

\section{Subjects}

This study recruited 611 parents, 534 of whom completed the study and were enrolled in the study (sampling process is shown in figure 1). Of those, 266 parents had a child with a DSM-5-defined diagnosis (referred as patient group) of ADHD $(n=107)$ or ASD $(n=159)$, and 268 parents with a healthy child (referred as control group). The children in the patient group and control group were sex and age matched. All patients' parents were recruited from the outpatient clinic of the Psychiatry Department of a research academic hospital from January 2016 to May 2017. For patients with ADHD or ASD, the diagnosis was made according to the DSM- 5 by a child and adolescent psychiatrist after comprehensive medical record review and an interview with patients and their caregivers.

The majority of children in the control group were from a local primary school and a small portion of them were from the local community. Researchers (with a master's degree in medicine) reviewed a self-developed questionnaire (including demographics, developmental history and medical history) filled out by the control group's parents and collected further information from teachers and/or parents as necessary to ensure that all the control group children satisfied the inclusion criteria which included (1) not diagnosed with any psychiatric disorders previously or presently, (2) without any major medical conditions and (3) 1-14 years old.

\section{Assessment tools}

The Chinese version of CGSQ was a self-administered scale. Responses were rated on a Likert scale: "not at all" (1 point), "sometimes so" ( 2 points), "half of the time" ( 3 points), "most of the time" (4 points) and "always true" (5 points). All items in the scale are forward-scored, 


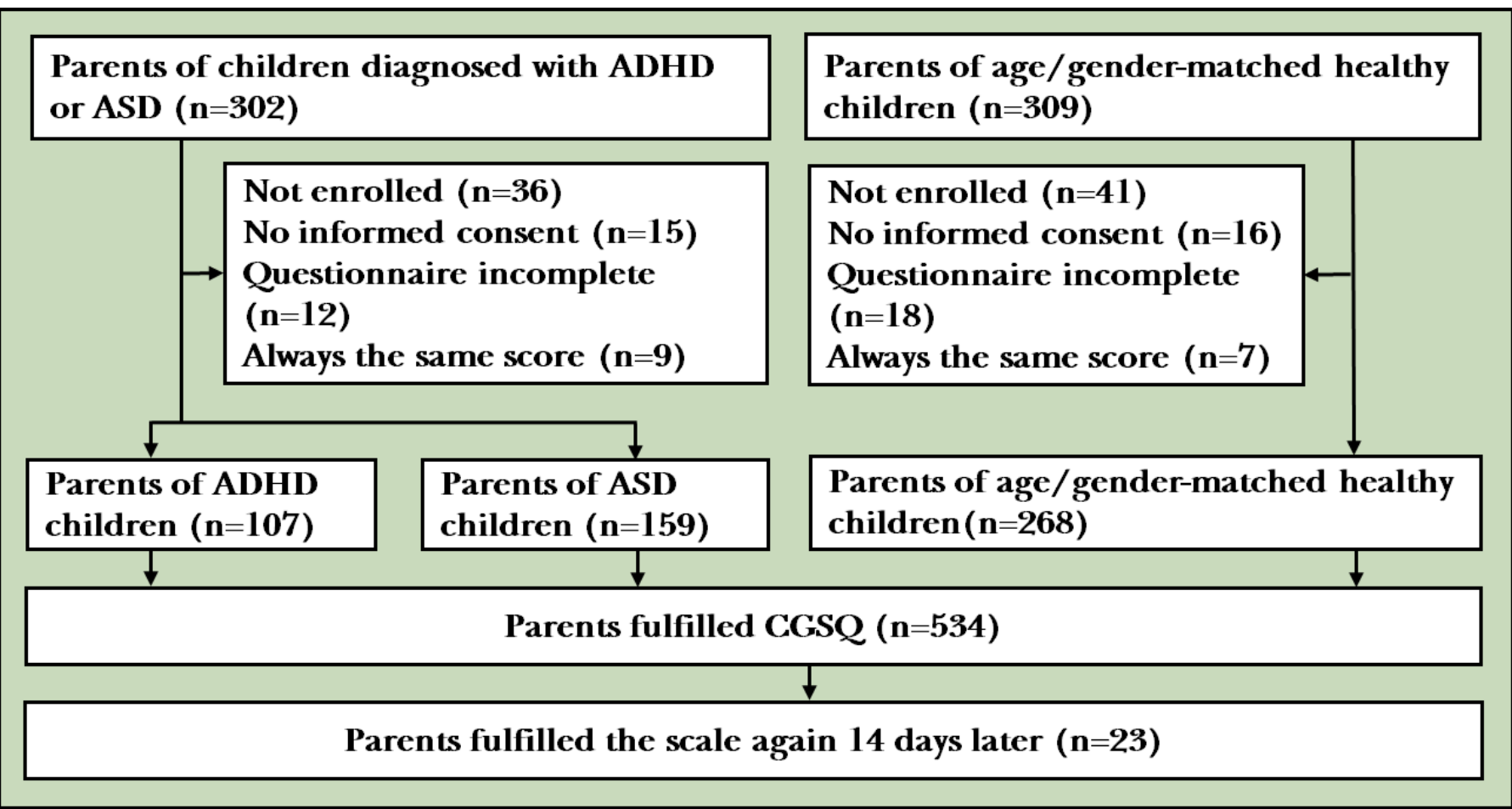

Figure 1 Flowchart of the study. ADHD, attention deficit hyperactivity disorder; ASD, autism spectrumdisorder; CGSQ, caregiver strain questionnaire.

and a higher total score indicates higher parenting stress. The total score of the scale ranges from 21 to 105 points. The Aberrant Behavior Checklist (ABC) was used for assessing problem behaviours of children with ASD. The ABC consists of 58 items resolving into five subscales which including irritability, social withdrawal, stereotypic behaviour, hyperactivity/noncompliance and inappropriate speech. The Conners Parent Symptom Questionnaire (PSQ) was used to evaluate symptoms of children with ADHD. The PSQ includes 48 items belonging to 6 subscales: conduct problem, learning problem, psychosomatic problem, impulsivityhyperactivity, anxiety and hyperactivity index. Higher total score indicates higher level of symptoms for the two scales mentioned above.

\section{Procedures}

The investigators were trained before recruiting participants. General demographic information (eg, gender, age, education) were acquired from all the parents and children with a self-developed questionnaire. Before conducting the CGSQ, the investigators first explained the scoring rules, then all the enrolled parents filled out the Chinese version of CGSQ; 14 days later, 23 subjects filled out the CGSQ again. At the data collection stage, investigators checked the CGSQ scale of each parent and excluded the parents filling in the CGSQ without following the scoring rules and those who did not finish the CGSQ completely. Epidata software was used for double data input, ensuring the input accuracy.

\section{Statistical analysis}

\section{Sample size estimation}

Tinsley ${ }^{19}$ recommended that sample size is 5 to 10 times of the number of variables, that is, there should be at least five times as many participants as variables in the factor analysis. Given that the CGSQ contains 21 items (variables), the sample size should be no less than 105 to meet the statistical requirements in each group.

\section{Description of the sample}

SPSS V.22.0 was used for organising data and comparing characteristics of participants between groups. We conducted independent-sample t-test to compare age and $\chi^{2}$ test to compare gender of participants between two groups. In order to describe the level of parenting stress, central tendencies (mean) and deviations (SD) were measured.

\section{Assessment of reliability of CGSQ}

After the factors (subscales) had been established, internal consistency was assessed for each subscale using SPSS V.22.0. The total scale Cronbach's $\alpha$ coefficient ${ }^{20}$ (Cronbach, 1951) is considered acceptable when greater than $0.70 .^{21} \mathrm{In}$ order to examine test-retest reliability of the instrument, the intra-class correlation coefficient (ICC) was calculated. ${ }^{22}$ ICC greater than 0.70 indicates sufficient test-retest reproducibility. ${ }^{23}$

\section{Exploratory factor analysis of CGSQ}

We randomly divided the patient sample into Group 1 $(\mathrm{n}=133)$ and Group $2(\mathrm{n}=133)$ and conducted EFA with 
Table 1 Characteristics of caregivers of control group and patient group

\begin{tabular}{|c|c|c|c|c|}
\hline & Control gr & 8) N (\%) & Patient grc & 6) N (\%) \\
\hline & Father & Mother & Father & Mother \\
\hline Age & & & & \\
\hline $20-30$ & $12(4.4)$ & $29(10.8)$ & 28 (10.5) & 44 (16.54) \\
\hline $31-40$ & $195(72.8)$ & $211(78.7)$ & $167(62.8)$ & $186(69.93)$ \\
\hline $41-60$ & $61(22.8)$ & $28(10.5)$ & 71 (26.9) & $36(13.53)$ \\
\hline Education level & & & & \\
\hline Middle school and below & $58(21.6)$ & $61(22.8)$ & $88(33.1)$ & $105(39.5)$ \\
\hline High school & $62(23.1)$ & $63(23.5)$ & $55(20.7)$ & $67(25.2)$ \\
\hline College & $121(45.2)$ & $122(45.5)$ & $108(40.6)$ & $87(32.7)$ \\
\hline Master/Doctoral & $27(10.1)$ & $22(8.2)$ & $15(5.6)$ & $7(2.6)$ \\
\hline Employment status & & & & \\
\hline Full-time & $162(60.4)$ & $110(41.0)$ & $130(48.9)$ & $87(32.7)$ \\
\hline Part-time & $26(9.7)$ & 65 (24.3) & $49(18.4)$ & $47(17.7)$ \\
\hline Other & 80 (29.9) & 93 (34.7) & 87 (32.7) & $132(49.6)$ \\
\hline
\end{tabular}

Group 1 of the patient sample and total control sample, using SPSS V.22.0. The suitability of factor analysis was verified based on the Kaiser-Meyer-Olkin (KMO) test and the Bartlett sphericity test. Principal component analysis (PCA) was applied to determine the factors of the CGSQ. Oblique rotation was chosen. Factors were determined on the basis of eigenvalues above 1.0 and observation of the scree plot.

\section{Confirmatory factor analysis of CGSQ}

To confirm the theoretical model, CFA was conducted with Group 2 of the patient sample and total control sample by the AMOS program, and least-squares estimation was chosen to estimate the parameters. The $\chi^{2}$ test, ${ }^{24}$ the root mean square error of approximation (RMSEA), the Goodness-of-Fit Index (GFI) and the Comparative Fit Index (CFI) were applied to evaluate the goodness-of-fit of the model. Goodness-of-fitis thought to be adequate when RMSEA $<0.05$ and acceptable when $<0.08$. When values of GFI and CFI exceed 0.90, goodness-of-fit is also thought to be adequate, and the higher the better. ${ }^{25}$

Comparisons of CGSQ scores among control group, ADHD and ASD To compare parents rating CGSQ scores of ADHD, ASD and control group children, one-way ANOVA was applied.
Post hoc analysis was then conducted to compare groups two-by-two according to Dunnett's method, with the mean difference significant at the 0.05 level. In order to describe the level of parenting stress, central tendencies (mean) and deviations (SD) were measured.

Influencing factors of parenting stress among control group, ADHD and ASD

Multiple linear regression analysis was conducted to identify the influencing factors of parenting stress in control group, ADHD group and ASD group, respectively. The CGSQ total score was used as the outcome variable. The ABC subscale scores, the subscale scores of PSQ and the demographic variables were taken as potential factors affecting parenting stress. All factors which were significant in the univariate analysis were put into the multivariate analysis. In multivariate analysis, $p$ value less than 0.05 was considered significant.

\section{RESULTS}

Description of the sample

Table 1 shows the characteristics of parents in the patient and control group. In the control group, mothers' age

Table 2 Comparisons of demographics between control group and patient group

\begin{tabular}{|c|c|c|c|c|}
\hline & \multirow{2}{*}{$\begin{array}{l}\text { Control group } \\
(n=268)\end{array}$} & \multirow{2}{*}{$\begin{array}{l}\text { Patient group } \\
(n=266)\end{array}$} & \multicolumn{2}{|c|}{ Analysis } \\
\hline & & & $\chi^{2}$ & $\mathbf{p}$ \\
\hline \multirow[t]{2}{*}{ Gender (male/female) } & $(232 / 36)$ & $(236 / 30)$ & 0.57 & 0.449 \\
\hline & N/mean (SD) & N/mean (SD) & $\mathbf{t}$ & $\mathbf{p}$ \\
\hline Age & $268 / 7.06(2.79)$ & $266 / 7.05$ (2.85) & 0.04 & 0.486 \\
\hline $1-6$ years & $98 / 4.08$ (1.31) & $110 / 4.28(1.32)$ & -1.10 & 0.387 \\
\hline $7-14$ years & $170 / 8.77(1.81)$ & 156/9.00 (1.85) & -1.12 & 0.983 \\
\hline
\end{tabular}


Table 3 Cronbach coefficient alpha of CGSQ and subscales for control group and patient group

\begin{tabular}{lllll}
\hline & Global scale & Subscale OCGS & Subscale ISCGS & Subscale ESCGS \\
\hline Control group & 0.901 & 0.877 & 0.775 & 0.698 \\
Patient group & 0.952 & 0.958 & 0.953 & 0.845 \\
\hline
\end{tabular}

CGSQ, Caregiver Strain Questionnaire; ESCGS, Externalized Subjective Caregiver Strain; ISCGS, Internalized Subjective Caregiver Strain; OCGS, Objective Caregiver Strain.

ranged from 25 to 46 years, with a mean age (SD) of 35 (4) years, and fathers' age ranged from 27 to 57 years, with a mean age (SD) of 38 (5) years. In the patient group, mothers' age ranged from 24 to 52 years, with a mean age $(\mathrm{SD})$ of $36(5)$ years, and fathers' age ranged from 25 to 50 years, with a mean age (SD) of $38(5)$ years. Most of the parents were aged between 31 and 40 years in both groups.

Table 2 shows the demographics of children in the patient and control group. For both groups, the majority of subjects were boys ranging from 1 to 14 years old, with a mean age (SD) of 7 (3) years. There was no significant difference between the two groups in age and sex.

\section{Assessment of reliability}

The Cronbach's alpha value of each subscale is listed in table 3. Cronbach's alpha of the total scale was 0.901 in the control group and 0.952 in the patient group. Testretest reliability of the total scale was 0.890 , and 0.917 for subscale OCGS, 0.629 for subscale ESCGS and 0.872 for subscale ISCGS (all $\mathrm{p}<0.001)$.

\section{Exploratory factor analysis}

The CGSQ data of the patient (Group 1) and the control groups both satisfied the suitability for PCA with a KMO measure value of 0.93 in the patient group and 0.91 in the control group. Both groups yielded a statistically significant result of Bartlett's Test of Sphericity (Field, 2005). PCA found three components for the patient group (figure 2) and four components for the control group (figure 3) with eigenvalues exceeding 1.0, which together explained $75.58 \%$ of the total variance for the patient

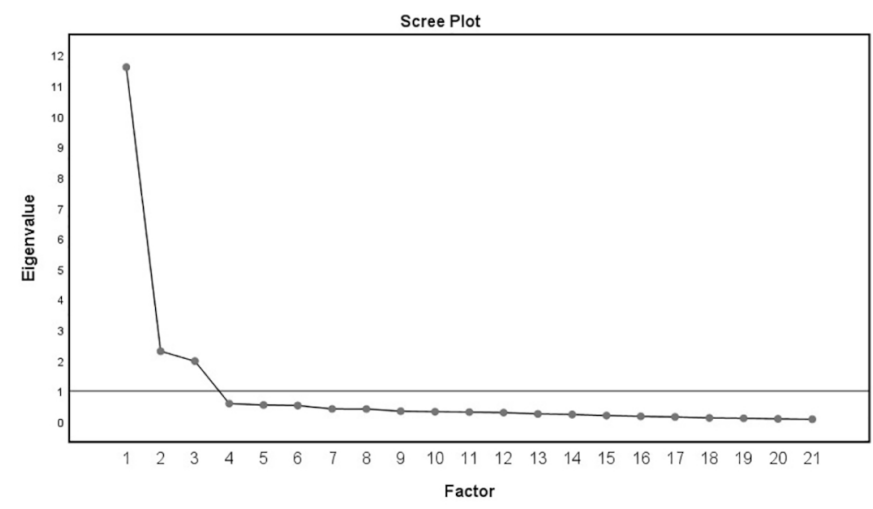

Figure 2 Scree plot of the CGSQ for patient group, representing the result of EFA. The horizontal axis indicates potential factor numbers, which determined by the Eigenvalue(the vertical axis), the final factor numbers of EFA is the Factor when the Eigenvalue $>1$. group and $62.44 \%$ for the control group. Furthermore, PCA also demonstrated that the items included in each dimension were consistent with the original proposal by Brannan in the patient group, with factor loadings ranging from 0.743 to 0.876 for each item (table 4).

\section{Confirmatory factor analysis}

For the patient group (Group 2), CFA revealed that the proposed three-factor model fit the data better $(\mathrm{CFI}=0.95$; GFI $=0.84$; RMSEA $=0.065$ ) compared with the two-factor model (CFI=0.86; GFI=0.73; RMSEA=0.11). However, for the control group, the fitting indices were not as good as those in the patient group for both the two-factor and three-factor models (table 5).

Comparisons of CGSQ total scores among control group, ADHD and ASD

The CGSQ total score of ASD group is statistically higher than that of both the control group and ADHD group in both 1-6 and 7-14 age groups (except for ASD vs ADHD in 1-6 age group). Likewise, The CGSQ total score of ADHD group is statistically higher than that of the control group in both 1-6 and 7-14 age groups (table 6).

Influencing factors of parenting stress among control group, ADHD and ASD

The multiple regression equations of CGSQ total score for normal controls, parents of a child with ADHD and parents of a child with ASD produced an adjusted $\mathrm{R}^{2}=0.042,0.183$ and 0.161 , respectively. In the control group, parents having a girl experienced a higher level of parenting stress than parents with a boy. In the ADHD

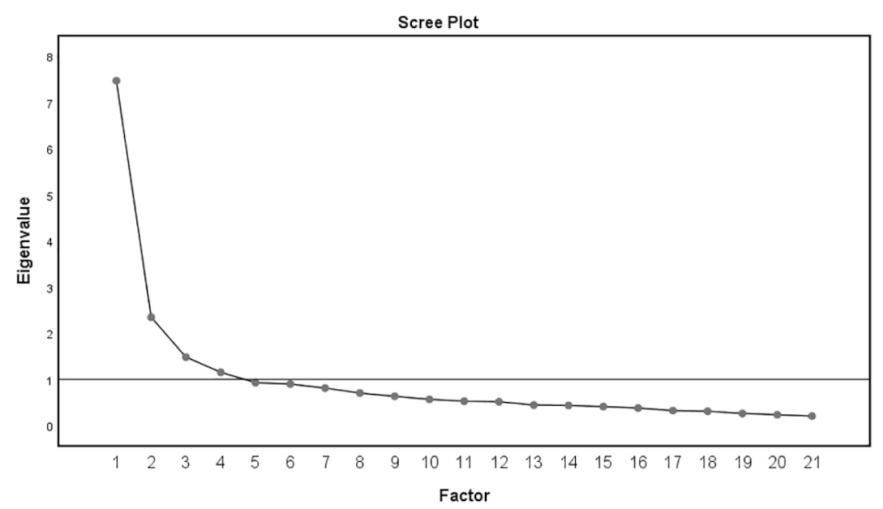

Figure 3 Scree plot of the CGSQ for control group, representing the result of EFA. The horizontal axis indicates potential factor numbers, which determined by the Eigenvalue(the vertical axis), the final factor numbers of EFA is the Factor when the Eigenvalue $>1$. 
Table 4 Principal component analyses of CGSQ for control group and patient group

\begin{tabular}{|c|c|c|c|c|c|c|c|}
\hline & \multicolumn{3}{|c|}{ Patient group } & \multicolumn{4}{|c|}{ Control group } \\
\hline & F1 & F2 & F3 & F1 & $\mathbf{F 2}$ & F3 & F4 \\
\hline Item 1 Personal Time Interrupted & 0.876 & & & 0.895 & & & \\
\hline Item 2 Missed Work, Neglect Duties & 0.784 & & & 0.816 & & & \\
\hline Item 3 Family Routines Disrupted & 0.823 & & & 0.693 & & & \\
\hline Item 4 Family Having to Do Without Things & 0.826 & & & 0.644 & & & \\
\hline Item 5 Family Suffering Negative Health Effects & 0.689 & & & & 0.667 & & \\
\hline Item 6 Child Getting into Trouble & 0.775 & & & & 0.633 & & \\
\hline Item 7 Financial Strain & 0.794 & & & & 0.662 & & \\
\hline Item 8 Less Attention to Family Members & 0.747 & & & & 0.522 & & \\
\hline Item 9 Disrupt Family Relationships & 0.801 & & & & 0.499 & & \\
\hline Item 10 Disrupt Family Social Activities & 0.785 & & & 0.676 & & & \\
\hline Item 11 Social Isolation & 0.824 & & & 0.503 & & & \\
\hline Item 12 Felt Sad or Unhappy & & 0.851 & & & 0.482 & & \\
\hline Item 13 Felt Embarrassed & & & 0.820 & & & 0.494 & \\
\hline Item 14 Relate well to Child* & & & 0.830 & & & 0.592 & \\
\hline Item 15 Angry towards Child & & & 0.761 & & & 0.778 & \\
\hline Item 16 Worried about Child Future & & 0.861 & & & & & 0.896 \\
\hline Item 17 Worried about Family Future & & 0.837 & & & & & 0.664 \\
\hline Item 18 Felt Guilty about child's illness & & 0.871 & & & & 0.706 & \\
\hline Item 19 Felt Resentful toward child & & & 0.770 & & & 0.792 & \\
\hline Item 20 Felt Tired or Strained & & 0.743 & & 0.509 & & & \\
\hline Item 21 Toll on Family & & 0.764 & & & 0.633 & & \\
\hline
\end{tabular}

${ }^{*}$ This item is the only reverse scoring item in the original scale, and it has been modified as forward scoring description in the Chinese version. CGSQ, Caregiver Strain Questionnaire.

group, better parental relationship and later onset of ADHD were indicative of a lower level of parenting stress. In the ASD group, more self-harm and stereotyped behaviours of children were indicative of a higher level of parenting stress (table 7 ).

\section{DISCUSSION}

High levels of parenting stress may result in parents' impatience, bad temper, and even verbal or physical abuse towards their children in extreme situations, which in turn undermines the parent-child relationship. ${ }^{26}{ }^{27}$ Studies demonstrate that high parenting stress will negatively affect parents' emotional communication with their children, causing a decline of parenting skills and negative parental behaviours. ${ }^{28}$ Caregivers with a higher level of stress are more likely to experience depression and anxiety, resulting in poor quality of life. ${ }^{29}$ Therefore, paying close attention to caregiver strain is of urgent concern in contemporary China.

CGSQ exhibited good construct validity and internal consistency reliability in several previous studies in different samples. ${ }^{14} 1518$ However, there is a paucity of studies that prove its feasibility in the Chinese population. Moreover, for the first time, the present study attempts to test psychometric properties of the CGSQ in typically developing children, considering that there is an urgent

Table 5 Fitting indices of two-factor model and three-factor model of CGSQ for control group and patient group

\begin{tabular}{lllllllllll}
\hline & & $\chi^{2} / \mathbf{d f}$ & RMSEA & RMR & GFI & NFI & RFI & IFI & TLI & CFI \\
\hline \multirow{2}{*}{ Control group } & Two-factor & 13.908 & 0.184 & 0.058 & 0.611 & 0.730 & 0.699 & 0.745 & 0.714 & 0.744 \\
& Three-factor & 4.169 & 0.109 & 0.039 & 0.776 & 0.708 & 0.670 & 0.761 & 0.728 & 0.759 \\
Patient group & Two-factor & 2.644 & 0.112 & 0.123 & 0.727 & 0.799 & 0.775 & 0.864 & 0.847 & 0.863 \\
& Three-factor & 1.564 & 0.065 & 0.068 & 0.841 & 0.882 & 0.867 & 0.954 & 0.948 & 0.954
\end{tabular}

CFI, Comparative Fit Index; CGSQ, Caregiver Strain Questionnaire; GFI, Goodness-of-Fit Index; IFI, Incremental Fit Index; NFI, Normed Fit Index; RFI, Relative Fit Index; RMR, root mean square residual; RMSEA, root mean square error of approximation; TLI, Tucker-Lewis Index. 
Table 6 Comparisons of CGSQ total scores among control, ADHD and ASD groups

\begin{tabular}{|c|c|c|c|c|c|c|}
\hline & \multirow[b]{2}{*}{$\begin{array}{l}\text { Total subjects } \\
\text { Mean (SD) }\end{array}$} & \multirow[b]{2}{*}{$\begin{array}{l}1-6 \\
\text { years old Mean (SD) }\end{array}$} & \multirow[b]{2}{*}{$\begin{array}{l}7-14 \\
\text { years old Mean (SD) }\end{array}$} & \multicolumn{3}{|c|}{ Analysis $p$ value } \\
\hline & & & & $\begin{array}{l}1-6 \\
\text { years old }\end{array}$ & $\begin{array}{l}7-14 \\
\text { years old }\end{array}$ & $\begin{array}{l}\text { Total } \\
\text { subjects }\end{array}$ \\
\hline ADHD group & 47.5 (15.2) & $43.0(10.2)$ & $47.8(15.5)$ & $0.081 \mp$ & $0.019 \ddagger$ & $0.021 \mp$ \\
\hline ASD group & $54.2(19.3)$ & $53.2(19.7)$ & $56.0(18.6)$ & $<0.001$ & $<0.001$ & $<0.001$ \\
\hline
\end{tabular}

${ }^{*}$ Control group vs ADHD group.

†Control group vs ASD group.

$\ddagger A D H D$ group vs ASD group.

ADHD, attention deficit hyperactivity disorder; ASD, autism spectrum disorder; CGSQ, Caregiver Strain Questionnaire.

need of such a tool for evaluating caregiver strain in the general population.

\section{Main findings}

Reliability refers to a scale's consistency and stability of testing results. ${ }^{30}$ In the patient group of this study, Cronbach's $\alpha$ coefficient was 0.952 for the CGSQ total scale, and from 0.845 to 0.958 for the three subscales (OCGS, ESCGS and ISCGS). However, it was notable that Cronbach's $\alpha$ of some of the proposed subscales, such as the ESCGS and ISCGS, were less impressive and under 0.80 in the control group. Moreover, our study also attained good test-retest reliability results for the Chinese version of the CGSQ.

Validity refers to the authenticity of the scale, that is, the degree to which psychological testing can detect certain psychological characteristics. ${ }^{30}$ Within the patient group, EFA revealed three factors with the same items in each dimension as the author originally designed. Furthermore, we conducted CFA based on the three-factor model and compared the results with the two-factor model simultaneously. Results showed that all the fit indicators of the three-factor model were better than those of the two-factor model. Put together, our results indicated that the three-factor model fit the Chinese population better.

Our study demonstrated acceptable validity, whether tested by EFA or CFA, and good reliability for the CGSQ in the patient sample. However, four factors were identified from EFA in the control group with scrambled items scattered among four different dimensions. Not surprisingly, following CFA revealed a poor fit to both the three-factor model and two-factor model within the control group. Considering this together with Cronbach's $\alpha$ coefficient, our study attained little support for the use of CGSQ in parents with a typically developing child. In regard to the differences in fit indicators between the patient and control groups, we speculate that some parents of the control group children might not take the administration of the CGSQ quite seriously and did not fill out the scale

Table 7 Linear regression analysis of influencing factors of parenting stress in caregivers of normal children and children with ASD or ADHD

\begin{tabular}{|c|c|c|c|c|c|}
\hline \multirow[b]{2}{*}{ Model } & \multicolumn{2}{|c|}{$\begin{array}{l}\text { Unstandardised } \\
\text { coefficients }\end{array}$} & \multicolumn{2}{|c|}{ Standardised coefficients } & \multirow[b]{2}{*}{$P$ value } \\
\hline & $\boldsymbol{\beta}$ & SE & $\beta$ & $\mathbf{t}$ & \\
\hline (Constant) & 35.662 & 3.912 & & 9.116 & $<0.001$ \\
\hline Sex & -2.980 & 1.412 & -0.131 & -2.110 & 0.036 \\
\hline Relationship of parents & 2.410 & 0.947 & 0.231 & 2.546 & 0.012 \\
\hline Age of onset & -1.719 & 0.756 & -0.218 & -2.274 & 0.025 \\
\hline \multicolumn{6}{|l|}{ ASD } \\
\hline (Constant) & 45.745 & 9.043 & & 5.059 & $<0.001$ \\
\hline
\end{tabular}

Adjusted $\mathrm{R}^{2}$ for caregivers of normal children $=0.042$.

Adjusted $\mathrm{R}^{2}$ for caregivers of children with $\mathrm{ADHD}=0.183$.

Adjusted $\mathrm{R}^{2}$ for caregivers of children with $\mathrm{ASD}=0.161$.

*Severity was calculated by summing the scores of 3 items related to self-harm behaviour in the ABC scale.

$A B C$, Aberrant Behavior Checklist; ADHD, attention deficit hyperactivity disorder; ASD, autism disorder. 
carefully since they were not experiencing an emergent need for help in comparison with parents of children with a diagnosed mental illness.

As expected, our results showed that the CGSQ total scores successfully differentiate the level of caregiver strain among different population: parents of children with ASD experienced the highest level of caregiver strain, followed by parents of children with ADHD, and then parents with a typically developing child, whether in total participants or in two different age groups (1-6 and 7-14 years old). This finding was consistent with clinical impression and therefore proposed a good discrimination validity of CGSQ.

It is important to identify factors affecting parenting stress. Multiple linear regression revealed a few factors affecting parenting stress among three groups of populations, despite the weak effects. Contrary to the findings of another study, ${ }^{31}$ our study showed that caregivers experienced higher parenting stress when fostering a typically developing girl compared with fostering a boy, which suggested that the role of gender on parenting stress may change with the growth of offspring and be influenced by other factors. ${ }^{32}$ In the ADHD group, better parental relationship and later onset of disease were correlated with lower level of parenting stress, which is consistent with our clinical experiences. Previous research indicated that ADHD comorbid problems were significant influencing factors to parenting stress. ${ }^{33}$ Comorbid problems should be included in our future studies since we did not collect the information of this aspect in the present study. In the ASD group, caregivers experienced a higher level of stress when their children exhibited more self-harm or stereotyped behaviours. A previous study also supported our finding which assumed that the severity of ASD behaviour problems led to an increase in parenting stress. ${ }^{34}$

To our knowledge, this is the first study to examine the construct validity and reliability of the CGSQ in the Chinese population. Our results suggest that the CGSQ appears to be feasible to evaluate the burden of taking care of children among Chinese parents who raise a child with ASD or ADHD. Within the patient population, there is evidence that the three-factor solution to the structure of CGSQ is better than the two-factor proposal. However, there is little support for the CGSQ as a reliable, robust measure that can be applied to caregivers with a normal child. Additional research is needed to evaluate the validity of the CGSQ in the broad population of people with emotional and behavioural problems. Furthermore, efforts should be made to explore the feasibility of the CGSQ for normal populations in another independent sample or/and after modifying some text description of certain items, because there is an urgent need to evaluate the parenting stress in normal population.

\section{Limitations}

This study has potential limitations. First, this study was performed in a selected population with ADHD or ASD, which limited the generalisation of the findings. Second, we did not conduct criterion validity, which may limit the strength of the findings. Future work should focus on populations with other psychiatric disorders, and criterion validity should be conducted.

\section{Implications}

These findings provide initial evidence to support the construct validity and reliability of CGSQ as a parenting stress measurement tool for Chinese parents, especially for parents of children with ADHD or ASD. Effective assessment tools can help clinicians better assess parenting stress and thus help patients and their caregivers more effectively.

Author affiliations

${ }^{1}$ Psychiatry Department, Kunming Medical University First Affilliated Hospital, Kunming, Yunnan, China

${ }^{2}$ Department of Psychosomatic Medicine, Shanghai East Hospital, Tongji University School of Medicine, Shanghai, China

${ }^{3}$ Psychiatry Department, Peking University Sixth Hospital, Beijing, China

${ }^{4}$ Psychiatry Department, Kunming Medical University Second Hospital, Kunming, Yunnan, China

${ }^{5}$ Department of Clinical Psychology, The Second People's Hospital of Yunnan Province, Kunming, China

${ }^{6}$ Department of Science and Education, Kunming Medical University Second Hospital, Kunming, Yunnan, China

Acknowledgements The authors would like to thank Professor Jin Lu of the First Affiliated Hospital of Kunming Medical University for her theoretical contribution and help with the text editing. We are also most grateful to all the parents who made the study possible by filling in the questionnaire.

Contributors CK, JY, RY and $\mathrm{HZ}$ designed the study and conceptualised the article. JL and YW introduced the Caregiver Strain Questionnaire (CGSQ) to China and translated the English version of CGSQ into Chinese. YW, RL, DH, YZ and XC contributed to subject enrolment and clinical data collection. RY, HZ, JY and CK contributed to data analysis and manuscript writing. SY contributed to manuscript writing and editing. CK and JY reviewed and revised the manuscript. All authors contributed to and approved the final manuscript.

Funding This work was supported by the National Natural Science Foundation of China (Grant No. 81460218, 30900488) and National Key Technology Research and Development Program of the Ministry of Science and Technology of China (Grant No. 2015BAl13B01).

Competing interests None declared.

Patient consent for publication Parental/guardian consent obtained.

Ethics approval The Ethics Committee of First Affiliated Hospital of Kunming Medical University approved this study (2018L N0.3). The study procedure was in accordance with the principles of the Helsinki Declaration (World Medical Association, 2013).

Provenance and peer review Not commissioned; externally peer reviewed. Data availability statement Data are available on reasonable request.

Open access This is an open access article distributed in accordance with the Creative Commons Attribution Non Commercial (CC BY-NC 4.0) license, which permits others to distribute, remix, adapt, build upon this work non-commercially, and license their derivative works on different terms, provided the original work is properly cited, appropriate credit is given, any changes made indicated, and the use is non-commercial. See: http://creativecommons.org/licenses/by-nc/4.0/.

ORCID iD

Runxu Yang http://orcid.org/0000-0002-0367-1139

\section{REFERENCES}

1 Deater-Deckard K. Parenting stress and child adjustment: some old hypotheses and new questions. Clin Psychol 1998;5:314-32. 
2 Abidin RR. Parenting stress and the utilization of pediatric services. Child Health Care 1983;11:70-3.

3 Sheras P, Abidin R, Konold T. Stress index for parents of adolescents. Odessa: Psychological Assessment Resources, 1998.

4 Crnic K, Low C. Everyday stresses and parenting.. In: Mahwah $\mathrm{NJ}$, ed. Handbook of parenting. 2. London: Lawrence Erlbaum Associates, 2002.

5 Pisula E, Porębowicz-Dörsmann A. Family functioning, parenting stress and quality of life in mothers and fathers of Polish children with high functioning autism or Asperger syndrome. PLoS One 2017;12:e0186536.

6 Hastings RP. Child behaviour problems and partner mental health as correlates of stress in mothers and fathers of children with autism. $J$ Intellect Disabil Res 2003;47:231-7.

7 Yatchmenoff DK, Koren PE, Friesen BJ, et al. Enrichment and stress in families caring for a child with a serious emotional disorder. $J$ Child Fam Stud 1998;7:129-45.

8 Kazdin AE, Child KA. Child, parent and family dysfunction as predictors of outcome in cognitive-behavioral treatment of antisocial children. Behav Res Ther 1995;33:271-81.

9 Song Y. Losing an only child: the one-child policy and elderly care in China. Reprod Health Matters 2014;22:113-24.

10 Zheng Y, Lawson TR, Anderson Head B. "Our only child has died"-a study of bereaved older Chinese parents. OMEGA - Journal of Death and Dying 2017;74:410-25.

11 HSBC. The value of education, higher and higher. p1-38, 2017. Available: http://www.hsbc.ae/1/PA_ES_Content_Mgmt/content/uae_ pws/pdf/en/voe-report.pdf

12 Loyd BH, Abidin RR. Revision of the parenting stress index. J Pediatr Psychol 1985;10:169-77.

13 Johnson N, Frenn M, Feetham S, et al. Autism spectrum disorder: parenting stress, family functioning and health-related quality of life. Fam Syst Health 2011;29:232-52.

14 Brannan A, Heflinger C, Bickman L. The Caregiver Strain Questionnaire: measuring the impact on the family of living with a child with serious emotional disturbance. J Emot Behav 1997:5:212-22.

15 Khanna R, Madhavan SS, Smith MJ, et al. Psychometric properties of the Caregiver Strain Questionnaire (CGSQ) among caregivers of children with autism. Autism 2012;16:179-99.

16 Bussing R, Gary FA, Mason DM, et al. Child temperament, ADHD, and caregiver strain: exploring relationships in an epidemiological sample. J Am Acad Child Adolesc Psychiatry 2003;42:184-92.

17 Kang E, Brannan A, Heflinger C. Racial differences in reports of caregiver strain among caregivers of children with emotional and behavioral problems. J Child Fam Stud 2005;14:43-56.
18 Ds HL. Factor analysis of caregiver strain questionnaire in clinical research statistics and data analysis, 2008: 383-90.

19 Tinsley EAT D. Uses of factor analysis in counseling psychology research. J Clin Psychol 1987;34:414-24.

20 Cronbach LJ. Coefficient alpha and the internal structure of tests. Psychometrika 1951;16:297-334

21 Nunnally J. Psychometric theory. 3 ed. New York: McGraw-Hill, 1994.

22 Bartko JJ. The intraclass correlation coefficient as a measure of reliability. Psychol Rep 1966;19:3-11.

23 Weir JP. Quantifying test-retest reliability using the intraclass correlation coefficient and the SEM. J Strength Cond Res 2005;19:231-4.

24 Byrne B. Structural equation modeling in AMOS: basic concepts, applications and programming. New York: Routledge=Taylor \& Francis, 2010.

25 Bentler PM. Cutoff criteria for fit indexes in covariance structure analysis: conventional criteria versus new alternatives. Struct Equ Modeling 1999;6:1-55.

26 Kupriyanova TA, Dubinskaya AO, et al. [An influence of mental disorder in the child on the parents in the context of differentiated approaches to psychosocial interventions on childhood psychiatry] Zh Nevrol Psikhiatr Im S S Korsakova 2014;114:14-19.

27 Osborne LA, Reed P. Stress and self-perceived parenting behaviors of parents of children with autistic spectrum conditions. Res Autism Spectr Disord 2010;4:405-14.

28 Rodriguez CM, Green AJ. Parenting stress and anger expression as predictors of child abuse potential. Child Abuse Negl 1997;21:367-77.

29 Vadher S, Desai R, Panchal B, et al. Burden of care in caregivers of patients with alcohol use disorder and schizophrenia and its association with anxiety, depression and quality of life. Gen Psychiatr 2020;33:e100215.

30 Miles. Effect of the intensive care unit environment on patents cardiac surgery children maternal child. Nursing 2003;11:383.

31 Scher A, Sharabany R. Parenting anxiety and stress: does gender play a part at 3 months of age? J Genet Psychol 2005;166:203-14.

32 Gordon CT, Hinshaw SP. Parenting stress and youth symptoms among girls with and without ADHD. Parent Sci Pract 2017:17:11-29.

33 Muñoz-Silva A, Lago-Urbano R, Sanchez-Garcia M, et al. Child/ adolescent's ADHD and parenting stress: the mediating role of family impact and conduct problems. Front Psychol 2017;8:2252.

34 Rodriguez G, Hartley SL, Bolt D. Transactional relations between parenting stress and child autism symptoms and behavior problems. J Autism Dev Disord 2019;49:1887-98.

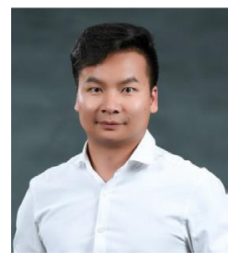

Runxu Yang obtained the master's degree in psychiatry from Kunming Medical University, China, in 2014. After graduating, he was trained in the department of psychiatry at the First Affiliated Hospital of Kunming Medical University, and since worked there until now. He participated as a researcher in a study about EOS, which was funded by the National Natural Science Foundation of China. He also has conducted research on $A D H D$ in the field of functional magnetic resonance imaging. His research interests include mental health issues among children and adolescents, especially attention deficit hyperactivity disorder (ADHD) and earlyonset schizophrenia (EOS). 